\title{
Article
}

\section{Exploring health care professionals views on alternative approaches to cancer follow-up and barriers and facilitators to implementation of a Recovery Package}

Williamson, Susan, Beaver, Kinta and Langton, Stephen

Available at http://clok.uclan.ac.uk/32884/

Williamson, Susan ORCID: 0000-0002-9635-4473, Beaver, Kinta ORCID: 00000002-6552-2323 and Langton, Stephen (2020) Exploring health care professionals views on alternative approaches to cancer follow-up and barriers and facilitators to implementation of a Recovery Package. European Journal of Oncology Nursing, 46 (10175). ISSN 1462-3889

It is advisable to refer to the publisher's version if you intend to cite from the work. http://dx.doi.org/10.1016/j.ejon.2020.101759

For more information about UCLan's research in this area go to http://www.uclan.ac.uk/researchgroups/ and search for <name of research Group>.

For information about Research generally at UCLan please go to http://www.uclan.ac.uk/research/

All outputs in CLoK are protected by Intellectual Property Rights law, including Copyright law. Copyright, IPR and Moral Rights for the works on this site are retained by the individual authors and/or other copyright owners. Terms and conditions for use of this material are defined in the policies page. 


\section{Exploring health care professionals views on alternative approaches to cancer follow-up and barriers and facilitators to implementation of a Recovery Package}

\section{Introduction}

Implementing alternative models for the provision of follow-up care for patients treated for cancer has been the focus of cancer care reform in the United Kingdom (UK) over the last decade (Macmillan Cancer Support, 2008; Department of Health, 2010; NHS Improvement, 2010; NCSI, 2013; NHS England, 2016). The traditional model of regular hospital outpatient appointments is costly to the UK's National Health Service (NHS) and is practically and financially unsustainable, given the increasing numbers of cancer survivors across Europe (Ferlay et al, 2013). Current follow-up practices do not meet cancer survivors' psychosocial and information needs, fostering reliance on healthcare professionals (HCPs) and hindering the ability to self-manage (DoH, 2010; Macmillan Cancer Support, 2008). There is no standardised practice across European countries to meet the needs of cancer survivors and more research is needed to promote the long-term well-being of patients diagnosed with cancer (Lagergren et al, 2019)

In the UK, a rapid review of service provision following cancer treatment concluded that addressing the needs of cancer survivors, particularly with the predicted increase in numbers, required new models of follow-up (NHS Improvement 2010). Studies of alternative models of follow-up including General Practitioner (GP) led, nurse-led, and patient-initiated have reported effectiveness and been well received by patients (Grunfeld et al, 1996; 1999; Moore et al, 2000; Brown, 2002; Murchie et al, 2010; Beaver et al, 2009; 2012; 2017) but have not been routinely adopted into practice at a national level. In 2013 the UK's National Cancer Survivorship Initiative (NCSI) recommended an integrated package of care (Recovery Package) that included: a structured Holistic Needs Assessment (HNA) that incorporates a care plan based on an assessment of physical, practical, emotional, spiritual and social needs, treatment summaries for patients and GP's, patient education and support events (health and wellbeing clinics) and a cancer care review in primary care. These elements of the Recovery Package were considered the foundation for achieving 
quality outcomes for cancer survivors (NCSI, 2013) with the expectation that it will be offered to each person affected by cancer by 2020. A report aimed at supporting commissioners and clinical networks to drive improved outcomes for patient centred care, outlines the need for risk stratified pathways of care (NHS England, 2016). Hence, individuals deemed at low-risk of recurrence and late effects (physical and psychosocial), should be encouraged toward supported self-management with timely re-access and/or remote monitoring (NHS England, 2016). Patients considered to have a medium or high risk of recurrence, complex care needs and/or a poor prognosis (e.g. being treated for palliation rather than with curative intent) would receive either planned co-ordinated care or care from specialist services.

Despite the drive towards increased self-management and the need for follow-up services to be better managed, it is still not clear how alternative models of care have been implemented or what barriers / facilitators have been identified in NHS institutions across the UK when introducing the Recovery Package.

\section{Aim}

This study aimed to explore the views of HCP's, NHS managers and commissioners of services on alternative strategies for follow-up care, and the perceived barriers and facilitators to implementation of the Recovery Package for patients who had completed treatment for cancer.

\section{Method}

As this study was exploratory, a qualitative approach was taken involving individual telephone interviews with study participants. Semi-structured interviews sought participants' views and experiences of key aspects of cancer care follow-up: sustainability of the traditional model of hospital outpatient follow-up; whether alternative models of cancer follow-up had been implemented; barriers and facilitators which contributed to implementation of new models; aspects of the NCSI Recovery Package (HNA, treatment summaries, health and wellbeing clinics, cancer care review) and its implementation. 


\section{Sample}

Participants included HCP's (Clinical Nurse Specialists (CNS), Lead Cancer Nurses, consultant surgeons, oncologists and GPs), commissioners of cancer services and NHS managers who had responsibility for providing follow-up care for cancer patients. Clinical nurse specialists are nurses with advanced nursing skills within a specialist clinical area. They co-ordinate patient care, and provide information and support for patients and their families. In cancer care, they are employed in a number of different specialist areas. Lead Cancer Nurses provide professional leadership to clinical teams. NHS managers co-ordinate the complex range of services provided by the NHS. In cancer care, NHS managers take a leading role on driving improvements in patient pathways and provide leadership and management support to clinical staff to ensure patients and their families receive high quality care and support. Commissioners of services in cancer are senior managers responsible for the planning, prioritisation, purchasing and monitoring of health care services. To ensure representation of views across the UK, participants from primary and secondary care, commissioning groups and national cancer centres were invited to participate. Participants were located in a diversity of geographical regions including Scotland, Wales, the Midlands, Yorkshire, the North West, South East and South West of England.

A combination of convenience and snowball sampling was utilised to identify participants. A study advisory group comprised individuals who had clinical, commissioning and managerial experience

\section{Access and Recruitment}

Potential participants were sent an introductory e-mail outlining the background, rationale and aims of the study. A participant information sheet and consent form were attached, inviting them to participate in an individual telephone interview, or face to face if preferred if they had more than one years' experience (clinically or managerially) providing follow-up services for patients who had been treated for cancer. Electronic agreement to participate served as confirmation of consent. All participants had experience of working in cancer care. A mutually agreed date and time for interview was arranged. Interviews were conducted by 
one researcher unknown to the participants. With consent, interviews were digitally recorded and subsequently transcribed.

Ethical approval was gained from the University of Central Lancashire in North West England.

\section{Analysis}

Interviews were transcribed verbatim and uploaded into MAXQDA, a software package for qualitative data management (www.maxqda.com). The data were independently reviewed and analysed using thematic analysis (Braun \& Clarke, 2006) by two of the authors who were not involved in the interviews. Each interview was read several times and coded into significant, recurrent themes and subthemes which reflected the meaning of the overall dataset. Any differences were resolved through discussion.

\section{Findings}

Twenty-one individuals agreed to participate; 19 interviews were conducted over the telephone and two face-to-face. Participants had been in their current post for a mean of 7 years (ranging from 1.5 to 18 years). The posts held by participants are presented in Table 1. Seven themes emerged from the data; sustainability and capacity, cost implications, primary care, self-management, Recovery Package, resistance to change, and visions for the future. (insert Table 1)

\section{Sustainability and capacity}

There was consensus that traditional, hospital-based follow-up for all patients following cancer treatment was unsustainable. Current services were struggling to accommodate the number of patients seen within clinical settings, due to increased incidences of cancer and survival rates.

Traditional hospital follow-up was considered suitable for patients with complex needs, rarer forms of cancer and/or a poorer prognosis. Risk stratification was considered a vital 
element of any future strategy but, if early detection of recurrence could lead to curative treatment, then hospital follow-up was considered important.

'So in certain tumour groups I would absolutely say it's very important that people are still monitored within the clinical team with that expertise... a very good example of that might be in something like anal cancer... it's probably very appropriate that that patient group is followed up within a hospital setting with the experts.' (ID 19 consultant nurse)

All participants reported that their institutions had attempted to implement alternative models of care, with varying degrees of progress and success. Most alternative models of follow-up were in the developmental or early phases of implementation, focussing on patients who had completed treatment for common cancers, such as breast and prostate.

Nurse-led approaches, particularly telephone follow-up, were common and had been in place for a number of years at some locations. Implementing telephone follow-up was considered reasonably straight forward, as it required replacing a face- to- face service with a telephone call and had widespread support. This mode of follow-up care was reported to suit patients who did not necessarily require a physical examination. However, telephone follow-up was viewed as a useful stop gap, not a long-term solution, as it was not possible to follow-up all cancer survivors in this way due to workforce issues and a lack of succession planning.

'They're [clinical nurse specialists] the key people and there are big workforce implications there because they don't grow on trees...so if you have more patients go into a nurse led follow-up well that releases your oncologist or your consultant, but you've got to have the nurses, they take time to learn and train up, so it, it's got to kind of happen at the pace at which people can skill up and come into it.' (ID 04 GP \& Commissioner)

Remote tracking was reported as particularly beneficial for patients whose follow-up care required regular scans or blood tests as results could be communicated and accessed via post or, in some cases, online. However, it was noted that flexibility would need to be built into community-based follow-up to ensure that patients could be easily recalled if there was cause for concern. 


\section{Cost implications}

The financial burden associated with traditional follow-up was a recurring theme, not only in terms of costs to the NHS, but also costs for patients. Costs to patients included the emotional costs of raised anxieties prior to appointments and financial costs in terms of travelling and time out of work.

'...we see patients who go for a scan, lie awake for two nights before their appointment, which is three weeks after their scan, worrying about has their cancer come back, go to the appointment, pay ... four pounds to park, wait an hour and a half to see the specialist who briefly says "oh your scan's fine that's lovely no problem, see you in a year".' (ID 04 GP \& Commissioner)

It was widely acknowledged that introducing new ways of working would require investment, but resources were limited. Some initiatives had been pump primed (a temporary investment of funds to stimulate development and implementation of elements of the recovery package) by charitable organisations (e.g. Macmillan Cancer Support, Prostate Cancer UK) but there was concern over how the initiatives could be sustained once the initial funding period ended. Resources needed to be available so that patients could be prioritised according to greatest need and highest risk of recurrence. However, there was concern that there was little incentive for NHS hospital Trusts (which provide secondary care) to give up hospital follow-up as they would lose their payment for follow-up appointments.

'Imagine it for each patient with breast cancer that the tariff for seeing somebody in outpatients is a hundred pounds, and there's two thousand patients that are seen four times a year ... but then imagine that disappears...it's a disincentive for acute Trusts to change....' (ID 14 GP \& Clinical Lead for Cancer)

Patient-initiated approaches would also require funding and resources. Participants argued that fewer hospital appointments did not equate to no follow-up as systems still needed to be in place to ensure effective monitoring. 
'I think the challenges have been to ensure that we have commissioning agreement, that we're paid. Just because we're not seeing the patients doesn't mean that we're not still following them up in as much as we are still organising all of the surveillance... I think that the challenge is, is helping the commissioners and the GPS understand that we're not just throwing patients out there and saying, get on with it, we are still doing the follow-up, but it's in a very different way,' (ID 08 Head of Cancer Nursing)

A service specification was viewed as a useful lever when implementing new models. The importance of communication with commissioners was strongly emphasised. Funding would still be required for any alternative approach and any new approach would need to be commissioned accordingly so that funding was not simply cut.

'The facilitating factors from our point of view is having a service specification that states very clearly we expect to see this stratified individual approach to follow-up and you've got that contractual lever then if it's in the service specification.' (ID 05 Cancer Commissioning Manager)

\section{Primary care}

Stronger links between secondary and primary care were advocated to enhance patient experiences of follow-up, particularly promotion of patient-initiated approaches. If patients were to be discharged back to primary care, then a link person was required to ensure patients knew who to contact with any concerns. While some participants felt this could be done by an administrator, others were adamant that a CNS should undertake this role. If primary care were to take on more responsibility for follow-up, they would need more specialist nurses to link between primary and secondary care. A clear distinction was expressed on the roles of specialist nurses in primary and secondary care, with recommendations that primary care nurses address long-term follow-up and hospital-based nurses address more acute needs of newly diagnosed patients.

There were cost implications for primary and secondary care if GP's had a more prominent role in follow-up. However, the GP's who participated indicated that they could carry out follow-up, especially for patients treated for prostate cancer who would require PSA testing and monitoring, but only if self-management approaches were promoted and commissioning was altered to reflect the change in service provider. GP's would not be able 
to add to their workload by providing follow-up services unless they were appropriately funded and trained. GP's would also need rapid access back to specialist services if they had any concerns, and training to ascertain the tests and investigations required for patients.

Participants expressed concern that patients discharged early may become 'lost' in the system unless 'safety nets' were in place so that patients and GP's knew who to contact regarding problems and systems for rapid access to secondary care services were in place. Despite concerns, there was a favourable view on primary care practitioners taking a more prominent role in follow-up as primary care practitioners see the patient in the context of their environment and overall health and may have more understanding of patients' individual needs and ability to self-manage.

\section{Self-management}

One of the criticisms aimed at the traditional model of hospital-based follow-up was that it was not 'patient-friendly' and gave 'artificial support'. The traditional model was perceived as offering a false sense of security which deterred patients from taking responsibility for their own health and being attentive to signs of recurrence between appointments.

'We do have patients who come back who had symptoms weeks ago but thought oh it's alright l've got an appointment coming up.' (ID 02 Lead Cancer Nurse)

Participants emphasised the importance of describing the pathway for alternative models of follow-up at the beginning of a patient's cancer journey to manage their expectations. HCP's could emphasise the need for patients to be more involved in managing their own care and triggering action if and when necessary.

It was perceived that patients associated the traditional model with trust, and trust would need to continue if alternate models were introduced. Patients currently expected to return to hospital clinics on a regular basis and to be given a reassuring message that all was well. Concern was expressed that patients may feel unsupported or isolated if they were discharged immediately after treatment with no support mechanisms in place. It was felt 
that patients needed signposting to services and resources that could be readily accessed if required.

'... you need to manage patients expectations around that being the sort of follow-up that they can expect... if you're not careful and you follow-up people up at sort of arm's length in that way, it might actually exacerbate the problem of people feeling isolated rather than improve it.' (ID 18 Survivorship Network Manager)

Supported self-management was advocated as a key component of cancer care generally, regardless of stage in the cancer pathway, and strongly favoured by participants, although variation in implementation activities was reported. However, concerns were expressed about older patients who may not understand the new self-management approaches to care.

'What I'm finding is with the, the older patient is that they struggle with that ownership being put back onto them 'cos they're used to the paternalistic approach... younger patients seem to accept it better' (ID 13 Consultant Nurse)

Prostate cancer was viewed as being particularly suitable for self-management approaches as well as cancers with a good prognosis and those with obvious signs and symptoms of recurrence. Rapid access back to specialist services needed to be in place and it could not be assumed that discharging people from hospital clinics would not require resources.

'I think the infrastructure that's currently in place certainly here isn't enough to support that [supported self-management] because if you're sending the patients off you've got to give them clear routes back in and have things in place that can support them and we've got a few plans for the future that, that are going to involve a considerable amount of investment and it's a bit of a leap of faith for people at the minute because it's not been done before...' (ID 17 Lead Cancer Nurse)

\section{Recovery Package}

Most participants were aware of the different elements of the Recovery Package (HNA, risk stratification, treatment summaries, health and well-being clinics, cancer care review) but different aspects were being implemented at different rates. It was acknowledged that implementing the full Recovery Package required significant change at a time of decreasing resources. 
Only one participant (ID11) reported on all aspects of the Recovery Package being implemented in secondary care, utilising risk stratification for breast cancer patients. Similar pathways had been written for colorectal and prostate cancer patients at this location with a pathway for skin cancer patients in progress. Nurse specialists had taken on extra workloads to sustain the programme and funding had been received from a charitable source. The challenges of sustaining the programme of work and changing traditional practices were acknowledged and effective communication with commissioners of services was highlighted as a facilitating factor.

'We're already having talks with our commissioners which are being led by our local cancer network to look at how we can jiggle the funds around really and commission the Recovery Package activity, but that would be on the proviso obviously that we reduce the follow-up 'cos there won't be more money and we've got a lot more patients coming in...' (ID 11 Lead Cancer Nurse)

Two participants had reported that a quality incentive payment, where funding is withheld until quality targets are achieved, may be useful in supporting the introduction of HNA's, care plans and treatment summaries to inform a cancer care review in primary care.

The Recovery Package was generally supported by participants, although considered to be useful in theory but not working efficiently and effectively in practice as yet. There was a willingness to implement aspects of the Recovery Package but uncertainly as to how this could be effective without additional support and resource. Adequate resources needed to be in place to create 'sustainable change' (ID18). There was concern about identifying patients' needs without the resources and services being in place to meet them.

'You know if we say okay, this person really has got quite severe psychological consequences of their cancer and treatment and they need a level 4 psychotherapist, we haven't got access to a level 4 psychotherapist...So it's all very well doing the needs assessment and it sounds, you know, it's great for the patient to say how they're feeling but if you can't address those needs, why find out if they have those needs in the first place?'(ID $21 \mathrm{GP})$ 
Treatment summaries were in place at a number of locations but concern was expressed that they were not always understandable to GP's and were written for the benefit of hospital doctors.

'Make them [treatment summaries] understandable to a GP you know, they're not written for GP's they're written for the hospital doctor so that when they see them again in clinic they understand them but they're not really written with primary care in mind.' (ID 20 GP)

There was variability as to whether the HNA was being implemented in practice, although it was not always referred to as an HNA. It was generally accepted that patients needed to have their needs assessed but there was a lack of clarity as to when this should be carried out and by who although most said it was carried out by nurses. Participants were unclear who would carry out the HNA if patients came to a regional cancer centre from a local hospital for treatment and then returned following treatment for follow-up care. There were stark differences reported between locations that had financial support from charitable organisations (e.g. Macmillan Cancer Support) and those that did not in carrying out HNA's.

'They're part of a Macmillan pilot, so their nurses now have an iPad with holistic needs assessment on, the patient has the iPad in the waiting room and does their own holistic needs assessment by putting their finger on the boxes. It magically goes up a net somewhere and comes back down so that when the patient comes through to the nurse it's come back down with all the information of holistic needs assessment has fallen into a care plan' (ID 04 GP \& Commissioner)

'For the breast patients, we didn't offer holistic needs assessments, unfortunately, early on and the clinical nurse specialists were over worked, had no capacity to do that, and whilst we have a support worker she can do some holistic needs assessment, but certainly not on every patient... (ID 08 Head of Cancer Nursing)

Those participants working in areas that had implemented health and wellbeing clinics had attempted to incorporate a range of different elements within their clinics to cater for patients differing needs. Interventions focused on educating patients and encouraging supported self-management and ranged from short 4-6 week courses to more prolonged 
interventions. Different terminology was often used, including 'hubs', 'market places', 'workshops', 'events', and the 'Coventry model' based on the HOPE (Helping Overcome Problems Effectively) courses which have been adopted by Macmillan Cancer Support and are freely available.

Health and well-being clinics needed to be tailored to different cancer types and were perceived as inappropriate for patients with a poor prognosis. There was a lack of clarity on the most appropriate time to offer such clinics and participants felt they were more likely to promote self-management if offered at an earlier stage. However, it was also reported that health and well-being clinics might not necessarily act as a replacement for hospital-based follow-up but could provide an additional support mechanism, with additional resource implications.

'What we found was that patients said it was too late in the pathway for health and well-being clinics; it was far too late at two years and they'd struggled for two years with needing some advice... it's an additional support for patients, ... we've had some really positive feedback, and I'm sure it does support them throughout the rest of the pathway, but it certainly doesn't reduce the number of visits that they have, so yeah that's been quite an interesting find along the way.' (ID 02 Lead Cancer Nurse)

Where funding was not readily available, participants reported working closely with established charities to ensure patients could access additional support and information. Although some health and wellbeing clinics had been primarily designed for cancer specific patient groups, their ability to offer support for generic issues faced by all cancer patients had enabled some clinics to offer the service to a wider range of patients. Participants voiced concern about being able to sustain clinics that had been implemented without additional funding.

\section{Resistance to change}

Participants acknowledged that changing the cultural view of follow-up may be problematic for both patients and HCP's. Medical consultants were often perceived as resistant to change, wanting to continue seeing patients in hospital clinics. However, it was 
acknowledged that the situation was highly complex and the differing needs of different types of cancer patients heightened the degree of complexity.

Resistance to minimalist approaches to follow-up was anticipated if a particular cancer type had a higher likelihood of recurrence (e.g. ovarian). The different personalities of service providers was also considered an issue in trying to implement change as was a general resistance to any new initiative.

'Unfortunately the NHS when challenged with change usually puts up a lot of resistance to try and facilitate or otherwise and we've experienced that with [nurse's name] telephone, this is a nurse specialist, her telephone clinic because we've had tremendous difficulties in getting appointments arranged on our patient management system which means she has to get her notes herself and sort out the follow-up and there's no tracking of patients that don't turn up so it's, it's, we could have people that are more adaptable to support the service but unfortunately we haven't had that.' (ID 15 Surgical Oncologist)

Clinicians welcomed a balanced range of patients in their clinics, including those who had complex problems and those who were well but returning for routine monitoring. Hence, clinicians many not want to 'let go' of some of their patients, particularly as some expressed concern regarding a potentially negative emotional impact on clinicians of only seeing those patients with complex problems.

The lack of evidence on the effectiveness of alternative strategies, particularly those that supported minimalist and patient-led approaches, was a concern. Evidence that was available could be ignored, with practitioners defaulting back to what was known and familiar. Waiting for evidence to become available was seen as a delaying factor and there was a strong impetus to implement change before the evidence was available.

'I think the evidence is there. I think the problem with many of these trials... is actually implementing it into practice afterwards because it's all well and good during the trial but then very rarely do people actually implement it afterwards.'(ID 16 Clinical Oncologist) 
Visons for the future

The survivorship agenda was perceived as a fairly recent initiative. It was recognised that large organisations had numerous priorities and agendas and survivorship was not a top priority at all locations. Achievable milestones were needed that could highlight progress. When participants discussed how cancer follow-up care could evolve in the future, it was widely regarded that technology could, and should, be better utilised. One participant suggested introducing more tele health-based appointments. Enabling patients to take control of their own information was suggested. Participants expressed surprise that technological advances ha d not been evident in follow-up care.

'I stopped writing notes twenty years ago when I first became a GP in 1996 ..., everything was entered onto the computer, why isn't that the case in hospitals, why isn't everything, and then you could just put it on a stick [memory stick/flash drive] and patients could carry their notes around on a stick.' (ID 21 GP)

There was a call for more evidence on the safety and effectiveness of new models and a need for a more standardised national approach with concerns expressed about the potential for litigation.

'Trusts run the risk of exposing themselves to, to potentially law cases further down the road, if we haven't got the evidence to say this is safe follow-up for these patients and this is what we're all agreeing to nationally. If you've got one hospital doing one thing and another hospital doing something else; if a patient from Hospital A gets a recurrence you know can we confirm or could we prove that if they'd been followed up the way Hospital B follows them up that it wouldn't have been picked up earlier and that's the concern I've got really.' (ID 17 Lead Cancer Nurse)

Participants acknowledged that changing the current format of follow-up cancer care would take time. However, one participant emphasised how 'small wins' and setting realistic and achievable milestones could still make a difference to patient's lives, in addition to keeping the subject of changing follow-up care prominent.

\section{Discussion}


This study achieved its aim to explore the views of HCP's, NHS managers and commissioners of services on alternative strategies for follow-up care, and the perceived barriers and facilitators to implementation of alternative approaches and/or the Recovery Package for patients who had completed treatment for cancer. Participants welcomed the survivorship agenda, implementing aspects that they could, with or without additional resources. The findings indicated that communication between primary and secondary care, and patients and healthcare providers could be improved. Accessing and sharing of information between primary and secondary care is an essential aspect of future implementation with more efficient use of technology. Digitisation of healthcare systems in the UK is ongoing. Although digital record keeping in primary care is widespread, the same does not apply to secondary care and the funding for development of separate systems in primary care and secondary care has not facilitated the creation of an integrated healthcare record (National Audit Office, 2013). Technological advances in healthcare are not as advanced as those in sectors such as banking and retail, which have aimed to improve the consumer experience and make services more accessible and convenient (Honeyman, Dunn, and McKenna 2016). The Wachter report (2016) recommends that interoperability of healthcare records between primary and secondary care across regions in the UK should be a priority for 2019 and that by 2022 national interoperability should be in place with penalties for those healthcare trusts who do not comply. Successful implementation of the Recovery Package and new approaches to follow-up provision are reliant on interoperability of healthcare records to enable effective communication and patient safety.

There is an abundance of evidence that alternative approaches to follow-up are effective, command high levels of patient satisfaction and low risk of undiagnosed recurrence (Grunfeld et al, 1996; 1999; Moore et al, 2000; Brown 2002; Murchie et al, 2010; Beaver et al, 2009; 2012; 2017; Siddika et al, 2015). However, participants reported concern that commissioning of care and funding continues to be based on national tariffs for face to face hospital follow-up (Davies \& Batehup, 2011; Frew et al, 2010; Jefford et al, 2013) in spite of the recommendations of the Living With and Beyond Cancer programme ( $\mathrm{DoH} 2013$ ). The lack of standardised approaches to alternative follow-up was a concern from a legal perspective for some participants. There was a perceived risk of litigation should an 
approach implemented in one Trust, but not in another, prove to be more effective for patients. These concerns are not without foundation as the NHS has seen a dramatic rise in litigation with the cost of pay-outs rising from $\mathrm{f28.6}$ billion in 2014-15 tof 55.4 billion in 2015-16 (CIPFA 2016). There were differing levels of concern expressed between sites that had funding from cancer charities and those that did not. Those participants fortunate enough to work in sites receiving pump priming funding from cancer charities were concerned about the ability to continue with initiatives such as HNA's and discharge summaries for all cancer patients once funding ceased. Whereas those who did not receive charitable funding were concerned about their ability to regularly complete a HNA with providing a HNA for all cancer patients seeming to be an impossible goal. This makes achieving the targets set out in the Achieving World-Class Cancer Outcomes strategy by 2020 seem unattainable (DoH 2015). The HNA is pivotal to the successful implementation of the Recovery Package (DoH 2013;2015) but there was a diversity of view on whether the HNA was formally used in practice. The CNS role seemed to be the lynchpin for all aspects of the Recovery Package, although there was concern that they were an over stretched resource. The UK's Cancer Patient Experience Survey (2014) reported that access to a CNS is the most important factor contributing to a positive patient experience and The Independent Cancer Taskforce (2015) supports participants views that not only are numbers of CNSs insufficient in many cancer specialities but their time is used inefficiently. Resources for referring patients for care that might be identified through a HNA were not available to all participants. This is concerning as Ahmed et al (2014) found that identifying patients' needs but not addressing them had a negative impact on patients. However, Health and Wellbeing clinics were viewed positively as a resource to supplement follow-up care and meet patients information needs although some participants felt that patients were frequently not referred at the right time for them.

Initiatives to support the implementation of the Recovery Package were often reliant on the goodwill of NHS staff, which is not sustainable. The UK's Achieving World-Class Cancer Outcomes Report (DoH, 2015) recommends that the Recovery Package and stratified care pathways be rolled out as quickly as possible. This is unlikely to happen outside of research 
studies and pilot initiatives until commissioning of flexible approaches to follow-up care is secured (Davies \& Batehup, 2011; Frew et al, 2010; Jefford et al, 2013).

\section{Limitations}

This was a small study, data collection was completed in 2015 and participants may give a different account of barriers and facilitators to implementing the Recovery Package if they were re-interviewed. However, Beaver et al (2019) reported that patients were not aware of key elements of the Recovery Package, namely the HNA, health and wellbeing clinics, cancer care reviews. Williamson et al, (2018) reported HNAs were not widely embedded into practice and specialist nurses were concerned that HNAs were becoming bureaucratic "tickbox exercises" not conducted at times which met patients' needs.

\section{Conclusion}

Only one site had implemented all aspects of the Recovery Package for patients with common cancers. Although charitable funding to pump prime initiatives had enabled progress to be made, the HNA was the only aspect of the recovery package that had been implemented for some patients in all sites.

We recommend that effective utilisation of CNS's and adequate training and support be provided for those staff expected to enable patients to self-manage and navigate further healthcare should they need it. Commissioning and appropriate funding of new approaches to follow-up that move the focus from secondary to primary care including rapid access back to secondary care is required. Effective communication between patients and primary and secondary would be facilitated by interoperability of health care records. Hence, engagement between commissioners and service providers is essential for a change in practice and long-term sustainability.

\section{References}

Ahmed, N., Ahmedzai, S.H., Collins, K., Noble B.2014. Holistic assessment of supportive and palliative care needs: the evidence for routine systematic questioning. BMJ Supportive \& Palliative Care 2014;0:1-9.

Beaver, K., Tysver-Robinson, D., Campbell, M., Twomey, M., Williamson, S., Hindley, A., Susnerwala, S., Dunn, G., Luker, K. 2009a. Comparing hospital and telephone follow-up after 
treatment for breast cancer: a randomised equivalence trial. British Medical Journal. 338; a3147.

Beaver, K., Campbell, M., Williamson, S., Procter, D., Sheridan, J., Heath, J., Susnerwala, S. 2012. An exploratory trial comparing telephone and hospital follow-up after treatment for colorectal cancer. Colorectal Disease. 14(10); 1201-1209.

Beaver, K., Williamson, S., Sutton, C., Hollingworth, W., Gardner, A., Allton, B., Abdel-Aty, A., Blackwood, K., Burns, S., Curwen, D., Ghani, R., Keating, P., Murray, S., Tomlinson, A., Walker, B., Willet, M., Wood, N., Martin-Hirsch, P. 2017. Comparing hospital and telephone follow-up for patients treated for Stage I endometrial cancer (ENDCAT Trial): a randomised, multicentre, non-inferiority trial. BJOG: an international journal of obstetrics \& gynaecology; 124(1):150-160

Beaver, K., Martin-Hirsch' P., Williamson, S., Kyrgiou, M., 2019. Exploring the acceptability and feasibility of patient initiated follow up for women treated for Stage I endometrial cancer. European Journal of Oncology Nursing. DOI: 10.1016/j.ejon.2019.101704

Braun, V., Clarke, V. 2006. Using thematic analysis in psychology. Qualitative Research in Psychology. 3 (2); 77-101.

Brown, L., Payne, S., Royle, G. 2002. Patient initiated follow-up of breast cancer. PsychoOncology. 11; 346-355.

CIPFA (2016) http://www.cipfa.org/cipfa-thinks/cipfa-thinks-articles/trends-andassumptions-the-nhs-litigation-authoritys-final-accounts (Accessed January 2018)

Davies, N.J., Batehup, L. 2011. Towards a personalised approach to aftercare: a review of cancer follow-up in the UK. J Cancer Surviv 5: 142-151.

Department of Health. 2010. National Cancer Survivorship Initiative Vision. Department of Health and Macmillan Cancer Support; London, UK

Department of Health. 2013. Living With and Beyond Cancer: Taking Action to Improve Outcomes

Department of Health. 2015. Achieving World-Class Cancer Outcomes. A Strategy for England 2015-2020. A report of the Independent Cancer Taskforce.

Department of Health and Social Care. 2016. Making IT work: harnessing the power of health information technology to improve care in England Wachter RM (chair)

Published 7 September 2016

Ferlay, J., Steliarova-Foucher, E., Lortet-Tieulent, J., Rosso, S., Coebergh, JW., Comber, H., Forman, D., Bray, F. 2013. Cancer incidence and mortality patterns in Europe: estimates for 40 countries in 2012. European Journal of Cancer 49, 1374-1403. 
Frew, G., Smith, A., Zutshi, B., Young, N., Aggarwal, A., Jones, P., Kockelbergh, R., Richards, M., Maher, E.J. 2010. Results of a quantitative survey to explore both perceptions of the purposes of follow-up and preferences for methods of follow-up delivery among service users, primary care practitioners and specialist clinicians after cancer treatment. Clin Oncol 22: 874-884.

Grunfeld, E., Mant, D., Yudkin, P., Adewuyi-Dalton, R., Cole, D., Stewart, J., Fitzpatrick, R., Vessey, M. 1996. Routine follow up of breast cancer in primary care: randomised trial. British Medical Journal. 313; 665-669.

Grunfeld, E., Gray, A., Mant, D., Yudkin, P., Adewuyi-Dalton, R., Coyle, D., Cole, D., Stewart, J., Fitzpatrick, R. 1999. Follow-up of breast cancer in primary care vs specialist care: results of an economic evaluation. British Journal of Cancer. 79(7/8); 1227-1233

Honeyman, M., Dunn, P. \& McKenna, H. 2016. A Digital NHS? An introduction to the digital agenda and plans for implementation. The Kings Fund: available at;

https://www.kingsfund.org.uk/sites/default/files/field/field_publication file/A digital_NHS Kings_Fund Sep 2016.pdf (Accessed June 2019)

Lagergren, P., Schandl, A., Aaronson, N.K., Adami, H.A., de Lorenzo, F., Denis, L., Faithfull, S., Liu, L., Meunier, F., Ulrish, C. on behalf of the European Academy of Cancer Sciences. 2019. Cancer survivorship: an integral part of Europe's research agenda. Molecular Oncology. 13; 624-635

Macmillan Cancer Support. 2008. Two Million Reasons: The Cancer Survivorship Agenda. Macmillan Cancer Support; London

Moore, S., Corner, J., Haviland, J., Wells, M., Salmon, E., Normand, C., Brada, M., O'Brien, M., Smith, I. 2002. Nurse led follow up and conventional medical follow up in management of patients with lung cancer: randomised trial. British Medical Journal. 325; 1145-1152.

Murchie, P., Nicolson, M.C., Hannaford, P.C., Raja, E.A., Lee, A.J., Campbell, N.C. 2010. Patient satisfaction with GP-led melanoma follow-up: a randomised controlled trial. British Journal of Cancer. 102; 1447-1455.

Siddika, A., Tolia-Shah, D., Pearson, T.E., Richardson, N.G., Ross, A.H. 2015. Remote surveillance after colorectal cancer surgery: an effective alternative to standard clinic-based follow-up. Colorectal Disease.17(10):870-875

SNHS Improvement. 2010. Rapid review of current service provision following cancer treatment. NHS Improvement; Leicester, UK 
Jefford, M., Rowland, M., Grunfeld, E., Richards, M., Maher, J., Glaser, A. 2013. Implementing improved post-treatment care for cancer survivors in England, with reflections from Australia, Canada and the USA. Br J Cancer. Jan 15; 108(1): 14-20.

Achieving World-Class Cancer Outcomes. A Strategy for England 2015-2020. A report of the Independent Cancer Taskforce July .2015.

National Audit Office. 2013. https://www.nao.org.uk/report/review-of-the-final-benefitsstatement-for-programmes-previously-managed-under-the-national-programme-for-it-inthe-nhs/ (Accessed June 2019)

National Cancer Survivorship Initiative. 2013. Living With and Beyond Cancer: Taking Action to Improve Outcomes. NCSI: London

NHS England. 2016. Implementing the cancer taskforce recommendations: Commissioning person centred care for people affected by cancer.

https://www.england.nhs.uk/wp-content/uploads/2016/04/cancer-guid-v1.pdf (Accessed July 2018)

NHS England, Cancer Patient Experience Survey. 2014. Quality Health.

https://www.england.nhs.uk/cancer/

Williamson, S., Hack, T., Bangee, M., Benedetto, V., Beaver, K. 2018. The Holistic Needs Assessment in Cancer Care: Identifying Barriers and Facilitators to Implementation in the UK and Canada. Psycho-oncology https://onlinelibrary.wiley.com/doi/epdf/10.1002/pon.4638

www.maxqda.com 
